\title{
Implications of a class of neutrino mass matrices with texture zeros for non-zero $\theta_{13}$
}

\author{
Sanjeev Kumar \\ Department of Physics and Astrophysics, University of Delhi, \\ Delhi -11000\%, INDIA. \\ email: skverma@physics.du.ac.in
}

\begin{abstract}
A class of neutrino mass matrices with texture zeros realizable using the group $Z_{3}$ within the framework of type (I+II) seesaw mechanism naturally admits a non-zero $\theta_{13}$ and allows for deviations from maximal mixing. The phenomenology of this model is reexamined in the light of recent hints for non-zero $\theta_{13}$.
\end{abstract}

The observation of neutrino oscillations has provided us important information about neutrino masses and mixings. It has been known for a long time from the studies of atmospheric and solar neutrinos that the mixing angle $\theta_{23}$ can be maximal while the mixing angle $\theta_{12}$ is large but well below the maximal value. The smallest mixing angle $\theta_{13}$ is allowed to take any value from zero to an upper bound given by the accelerator and reactor neutrino experiments. However, the global analysis of neutrino data has provided hints for a non-zero $\theta_{13}$ at a small statistical significance not exceeding $2 \sigma$ level [1, 2, 3. The first direct observational hint for a non-zero $\theta_{13}$ has come from the Tokai-to-Kamioka (T2K) experiment which has rejected $\theta_{13}=0$ at $2.5 \sigma$ [4 . Shortly after T2K results, the Main Injector Neutrino Oscillation Search (MINOS) experiment has disfavored $\theta_{13}=0$ at $1.5 \sigma[5]$. A recent global analysis taking into account the recent T2K and MINOS results provides an evidence for $\theta_{13}>0$ at a confidence level greater than $3 \sigma$ [6]. The observation of a non zero $\theta_{13}$ may further lead to the measurement of $\mathrm{CP}$ violation in the lepton sector enriching our understanding of fermion mass generation and $\mathrm{CP}$ violation.

Many successful phenomenological patterns for the neutrino mass matrix have been postulated which predict $\theta_{13}=0$ as a natural prediction without any fine tuning of parameters. These ansatze or symmetries for the neutrino mass matrix force certain relations on the elements of neutrino mass matrix leading to increase in predictability and testability. Some popular examples of such ansatze are $\mu-\tau$ symmetry [7, 8, 9, 10, TBM mixing [1] and scaling [12. All these phenomenological patterns follow from some discrete flavor symmetries within the context of see-saw mechanism. These mass models can explain small non-zero values of $\theta_{13}$ as corrections or deviations from the exact symmetry. However, it may not be 
possible to accommodate sufficiently large value of $\theta_{13}$ in this way. If the recent hints for a large non-zero $\theta_{13}$ are confirmed in future, many such ansatze of neutrino mass matrix will be overruled. Even if a model survives this experimental test, one may need to overstretch the model parameters in order to get a sufficiently large $\theta_{13}$.

Another important phenomenological pattern is neutrino mass matrix with texture zeros [13] which is also realizable from discrete abelian flavor symmetries within the context of see-saw mechanism. There are seven patterns of texture zeros, further divided into three classes (A, $\mathrm{B}$ and $\mathrm{C}$ ), which are consistent with the present experimental data. The phenomenology of these classes of neutrino mass matrices has already been studied in details [14, 15] (and references cited therein). The main emphasis of the above studies was on finding the allowed parameter space and correlations among mixing angles and $\mathrm{CP}$ violating phases by restricting $\theta_{13}$ less than 6 degrees as required by the $\mathrm{CHOOZ}$ bound at $1 \sigma$ [14, 15. It was found that the classes $\mathrm{B}$ and $\mathrm{C}$ can give a large $\theta_{13}$ only for nearly maximal $\mathrm{CP}$ violation. This conclusion should not change even after the T2K results which point out to larger values of $\theta_{13}$. However, the restriction of $\theta_{13}<6$ degrees selects only a small portion of the full parameter space and provides only a partial understanding of the phenomenology of class A. The aim of present communication is to explore the phenomenology of class A for non-zero $\theta_{13}$ in the light of recent T2K and MINOS results.

It is possible to have the symmetry realization of this class of two texture zero mass matrices in the context of type (I+II) seesaw using the family symmetry $Z_{3}$ without assuming the dominance of either type of contributions keeping only the Standard Model scalar doublet which transforms trivially under the family symmetry thus effectively suppressing the undesired flavor changing neutral currents. An additional advantage of a single scalar doublet is the stability of zero textures in the neutrino mass matrix under renormalization group evolution. These texture zeros are enforced by extending the SM with one scalar SU(2) triplet. A complex scalar singlet which transforms nontrivially under the family symmetry is also introduced to get correct type I seesaw contribution [16].

The neutrino mass matrix $M$ in the flavor basis where the charged lepton mass matrix is diagonal can be parametrized in terms of neutrino masses $\left(m_{1}, m_{2}\right.$ and $\left.m_{3}\right)$, mixing angles $\left(\theta_{12}, \theta_{23}\right.$ and $\left.\theta_{13}\right)$ and $\mathrm{CP}$ violating phases $(\alpha, \beta$ and $\delta)$ as

$$
M=V M_{\nu}^{\operatorname{diag}} V^{T}
$$

where $M_{\nu}^{\text {diag }}=\operatorname{Diag}\left\{m_{1}, m_{2}, m_{3}\right\}$. The mixing matrix $V$ is given as

$$
V=\left(\begin{array}{ccc}
c_{12} c_{13} & s_{12} c_{13} & s_{13} e^{-i \delta} \\
-s_{12} c_{23}-c_{12} s_{23} s_{13} e^{i \delta} & c_{12} c_{23}-s_{12} s_{23} s_{13} e^{i \delta} & s_{23} c_{13} \\
s_{12} s_{23}-c_{12} c_{23} s_{13} e^{i \delta} & -c_{12} s_{23}-s_{12} c_{23} s_{13} e^{i \delta} & c_{23} c_{13}
\end{array}\right)\left(\begin{array}{ccc}
1 & 0 & 0 \\
0 & e^{i \alpha} & 0 \\
0 & 0 & e^{i(\beta+\delta)}
\end{array}\right),
$$

where $s_{i j}=\sin \theta_{i j}$ and $c_{i j}=\cos \theta_{i j}$. The class A of neutrino mass matrices with two texture zeros has two sub-categories: $A_{1}$ and $A_{2}$. In class $A_{1}, M_{e e}=0$ and $M_{e \mu}=0$ and in class $A_{2}$, $M_{e e}=0$ and $M_{e \tau}=0$ [13].

The existence of two texture zeros in the neutrino mass matrix give four constraints on the neutrino mixing parameters. These constraints can be rewritten in the form of two 
predictions on the mixing angles $\theta_{13}$ and $\theta_{23}$ and two predictions on the $\mathrm{CP}$ violating phases $\beta$ and $\delta$ in terms of the two unknown parameters $m_{1}$ and $\alpha$ [14] using the experimental values [6] of $\theta_{12}$ and two mass squared differences $\Delta m_{12}^{2}$ and $\Delta m_{23}^{2}$ which are used to express $m_{2}$ and $m_{3}$ in terms of $m_{1}$.

The predictions for the two mixing angles in class $A_{1}$ are [14]

$$
\tan ^{2} \theta_{13}=\frac{M}{m_{3}}
$$

and

$$
\tan ^{2} \theta_{23}=\frac{\mu_{3}^{2} \sin ^{2} 2 \theta_{12}}{4 M\left(M+m_{3}\right)}
$$

where

$$
M=\sqrt{m_{1}^{2} c_{12}^{4}+m_{2}^{2} s_{12}^{4}+2 m_{1} m_{2} c_{12}^{2} s_{12}^{2} \cos 2 \alpha}
$$

and

$$
\mu_{3}=\sqrt{m_{1}^{2}+m_{2}^{2}-2 m_{1} m_{2} \cos 2 \alpha} .
$$

The mixing angle $\theta_{13}$ is same in class $A_{1}$ and class $A_{2}$. However, the value of $\theta_{23}$ in class $A_{2}$ is given by [14]

$$
\tan ^{2} \theta_{23}=\frac{4 M\left(M+m_{3}\right)}{\mu_{3}^{2} \sin ^{2} 2 \theta_{12}} .
$$

The element $m_{e e}$ can only be zero for normal ordering of neutrino mass spectrum [17]. Therefor, the lightest neutrino mass is $m_{1}$ which is considered as a free parameter whereas $m_{2}$ and $m_{3}$ are expressed in terms of $m_{1}, \Delta m_{12}^{2}$ and $\Delta m_{23}^{2}$.

The predicted values of $\theta_{23}$ and $\theta_{13}$ span a bounded region on the $\left(\theta_{23}, \theta_{13}\right)$ parameter space depicted in Fig. 1. The dashed (red) line corresponding to $\alpha=0$ and the dotted (blue) line corresponding to $\alpha=90$ degrees form the lower boundaries of the region while the line $m_{1}=0.1 \mathrm{eV}$ forms the upper boundary of the region. This bounded region is in a way natural prediction of this class of texture zeros as no fine tuning of the parameters have been done till now. The experimentally allowed values of $\theta_{23}$ and $\theta_{13}$ [6] at $3 \sigma$ C.L. are shown as the solid contours. The predictions for classes $A_{1}$ and $A_{2}$ are mirror images of one another and are related through the transformation $\theta_{23} \rightarrow \pi / 2-\theta_{23}$. It can be seen from Fig. 1 that $\theta_{13}$ can be arbitrarily large irrespective of $\alpha$ in class $A_{1}\left(A_{2}\right)$ if $\theta_{23}$ is below (above) its maximal value. The only constraint which bounds $\theta_{13}$ from above in this region is an upper bound on the overall neutrino mass scale which has been arbitrarily fixed here at $m_{1}=0.1 \mathrm{eV}$ for the sake of illustration. However, the dotted line corresponding to $\alpha=90$ degrees forms both lower and upper bounds on $\theta_{13}$ in the region where $\theta_{23}$ is above (below) maximality in class $A_{1}\left(A_{2}\right)$. Moreover, the vanishingly small values of $\theta_{13}$ are unnatural in the sense that for these values the deviations of $\theta_{23}$ from maximality become too large. A non-zero value of $\theta_{13}$ consistent with the latest neutrino data [6] corresponds to nearly maximal $\theta_{23}$ and, therefore, is a natural prediction of this class of texture zero mass models. The above conclusions become more apparent in Figs. 2 and 3 where the contours of constant $\theta_{13}$ and $\theta_{23}$ have been plotted on the $\left(\alpha, m_{1}\right)$ parameter space. It is implicit from these figures that the innermost region where deviations of $\theta_{23}$ from maximal mixing becomes too large corresponds to $\theta_{13}<6$ degrees and, therefore, is disfavored. 
The above analysis has been done for the central values of $\theta_{12}, \Delta m_{12}^{2}$ and $\Delta m_{13}^{2}$. If the errors in these parameters are taken into account, the small values of $\theta_{13}$ become marginally allowed at $3 \sigma$. This can be seen by calculating $\theta_{13}$ and $\theta_{23}$ as functions of $m_{1}$ for $\alpha=90$ degrees by randomly sampling $\theta_{12}, \Delta m_{12}^{2}$ and $\Delta m_{13}^{2}$ [6]. The resulting plots have been depicted in Figs. 4 and 5. The central (red) regions show predictions for the mixing angles at $1 \sigma$ while the outer (green) regions give the $3 \sigma$ predictions. The experimental values [6] at $3 \sigma$ have also been depicted for comparison as the horizontal lines. It is evident from from Fig. 4 that $\theta_{13}$ becomes zero for $\alpha=90$ degrees at $3 \sigma$. Moreover, $\theta_{13}$ is non-zero at $1 \sigma$ and a considerable overlap exits between the model predictions and the experimental values for $\alpha=90$ degrees.

Figs. 3 and 5 also depict the complementarity between classes $A_{1}$ and $A_{2}$ in their predictions for $\theta_{23}$ in the sense that the predictions of one class are related to the predictions of the other class by the transformation $\theta_{23} \rightarrow \pi / 2-\theta_{23}$.

It has been shown in one of the earlier analyses [14] that predictions of classes $A_{1}$ and $A_{2}$ for $\theta_{23}$ differ at $3 \sigma$ if $\theta_{13}<5$ degrees. In such a case, $\theta_{23}$ will be above maximal in class $A_{1}$ and below maximal in class $A_{2}$ [14]. Since the new lower bound on $\theta_{13}$ at $3 \sigma$ is about 4 degrees [6], such a distinction between classes $A_{1}$ and $A_{2}$ cannot be made using the latest experimental results. The earlier experimental data allowed for this distinction between classes $A_{1}$ and $A_{2}$ as there was no lower bound on $\theta_{13}$.

In conclusion, it has been shown that $\theta_{13}=0$ is disfavored for neutrino mass matrices of class A since this requires $\alpha$ to be fine tuned near 90 degrees which leads to large deviations from the maximal value of $\theta_{23}$. If $\theta_{23}$ is below (above) maximal in class $A_{1}\left(A_{2}\right), \theta_{13}$ can be arbitrarily large as it is bounded from above only by the upper bound on absolute neutrino mass scale. Therefore, large values of $\theta_{13}$ can arise naturally in this class of neutrino mass models. Moreover, it is no longer possible to restrict $\theta_{23}$ to above maximality in class $A_{1}$ and below maximality in class $A_{2}$ which was the case when there were no lower bounds on $\theta_{13}$.

\section{References}

[1] G. L. Fogli et al., Phys. Rev. Lett. 101, 141801 (2008).

[2] M. C. Gonzalez-Garcia, M. Maltoni and J. Salvodo, JHEP 04056 (2010).

[3] T. Schwetz, M. Tortola and J. W. F. Valle, New J. Phys. 13063004 (2011).

[4] K. Abe et al [T2K Collaboration], arXiv:1106.2822 [hep-ex].

[5] L. Whitehead et al. [ MINOS Collaboration], Joint Experimental-Theoretical Seminar, (24 June 2011, Fermilab, USA).

[6] G. L. Fogli et al., arXiv:1106.6028 [hep-ph].

[7] T. Fukuyama and H. Nishiura, hep-ph/9702253.

[8] R. N. Mohapatra and S. Nussinov, Phys. Rev. D 60, 013002 (1999). 
[9] E. Ma and M. Raidal, Phys. Rev. Lett. 87, 011802 (2001).

[10] C. S. Lam, Phys. Lett. B 507, 214 (2001).

[11] P. F. Harrison, D. H. Perkins and W. G. Scott, Phys. Lett. B 530, 167 (2002).

[12] Anjan S. Joshipura and Werner Rodejohan, Phys. Lett. B 678, 276 (2009).

[13] Paul H. Frampton, Sheldon L. Glashow and Danny Marfatia, Phys. Lett. B 536, 79 (2002).

[14] S. Dev, Sanjeev Kumar, Surender Verma and Shivani Gupta, Nucl. Phys. B 784, 103 (2007).

[15] S. Dev, Sanjeev Kumar, Surender Verma and Shivani Gupta, Phys. Rev. D 76, 013002 (2007).

[16] S. Dev, Shivani Gupta and Radha Raman Gautam, Phys. Lett. B. 701, 605 (2011).

[17] S. Dev and Sanjeev Kumar, Mod. Phys. Lett. A 22, 1401 (2007). 
(a)

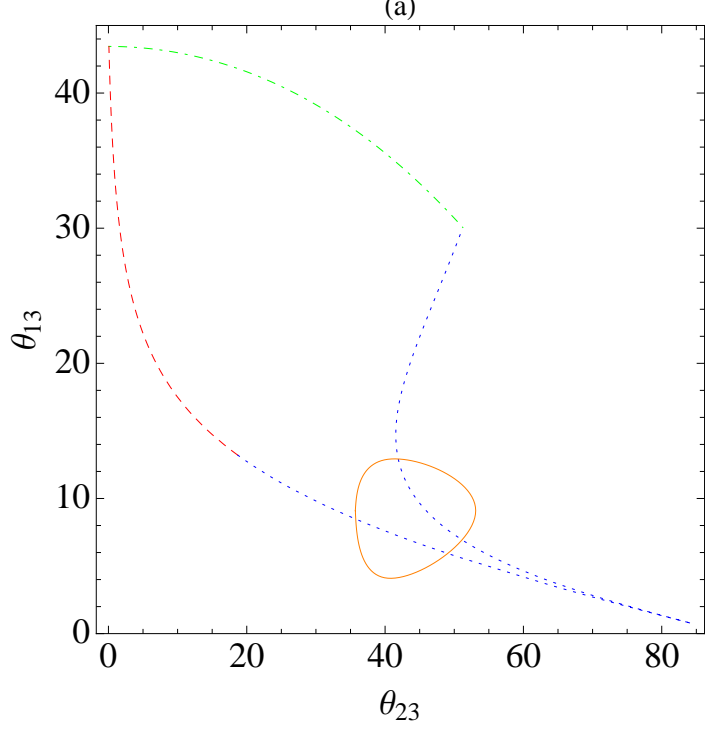

(b)

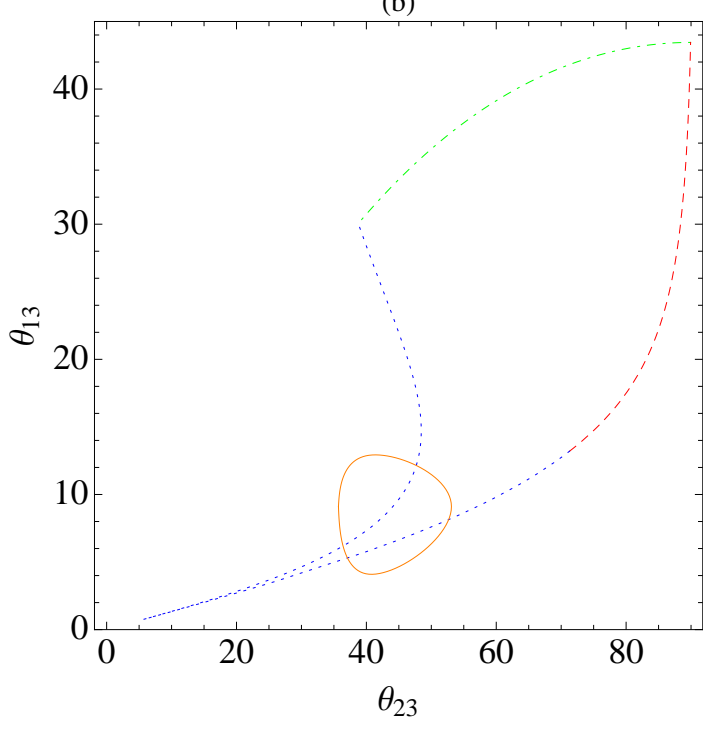

Figure 1: The regions spanned by predicted values of $\theta_{23}$ and $\theta_{13}$ for classes (a) $A_{1}$ and (b) $A_{2}$. The dashed (red) line corresponds to $\alpha=0$, the dotted (blue) line corresponds to $\alpha=90$ degrees and the upper dot-dashed (green) line corresponds to $m_{1}=0.1 \mathrm{eV}$. The solid (orange) contour shows the $3 \sigma$ region allowed by present experimental data.

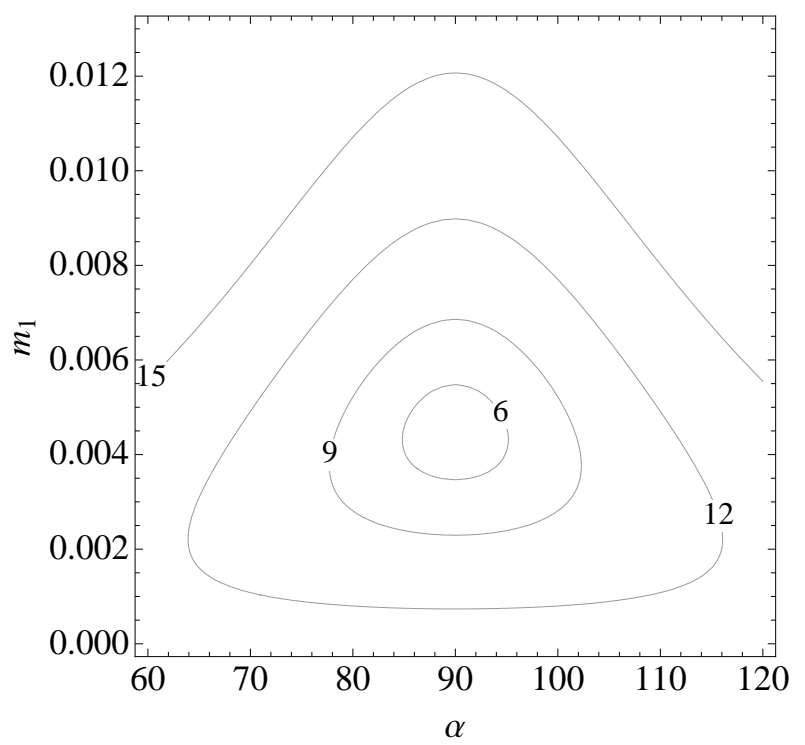

Figure 2: The contours for constant $\theta_{13}$ on $\left(\alpha, m_{1}\right)$ plane. 
(a)

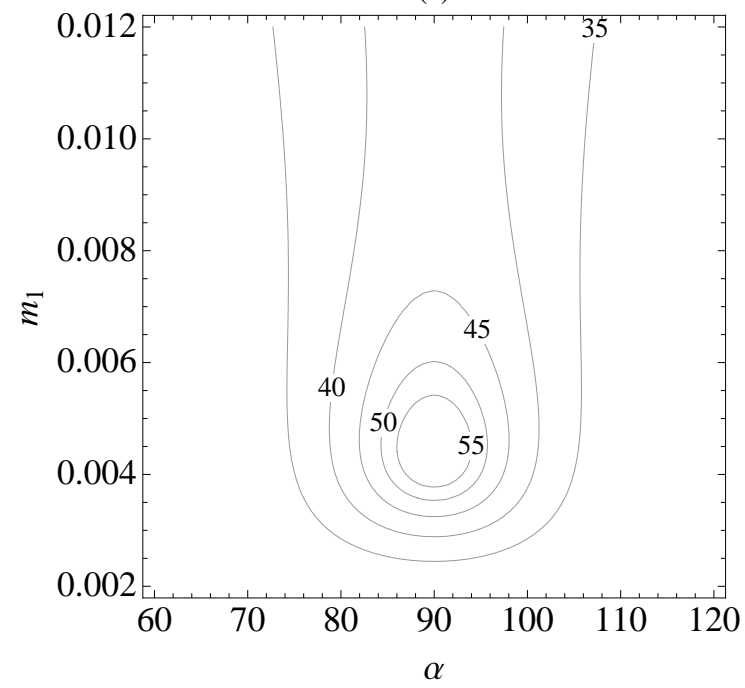

(b)

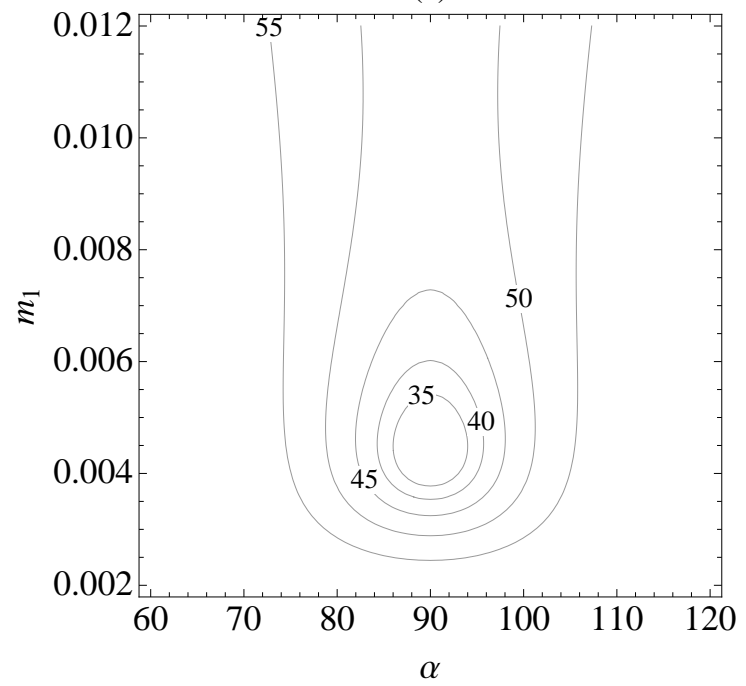

Figure 3: The contours for constant $\theta_{23}$ on $\left(\alpha, m_{1}\right)$ plane for (a) class $A_{1}$ and (b) class $A_{2}$.

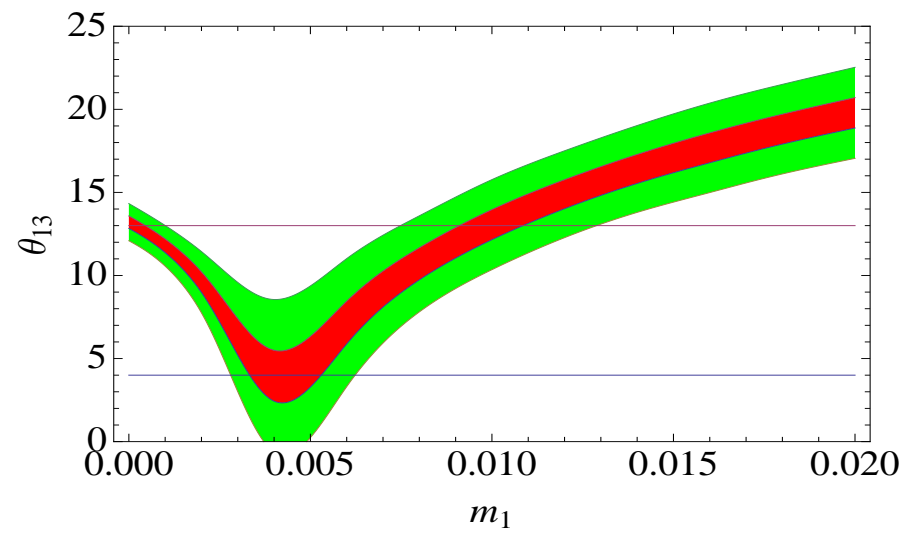

Figure 4: The variation of $\theta_{13}$ with $m_{1}$ for $\alpha=90$ degree. The central (red) region is at $1 \sigma$ and the outer (green) region is at $3 \sigma$. 
(a)

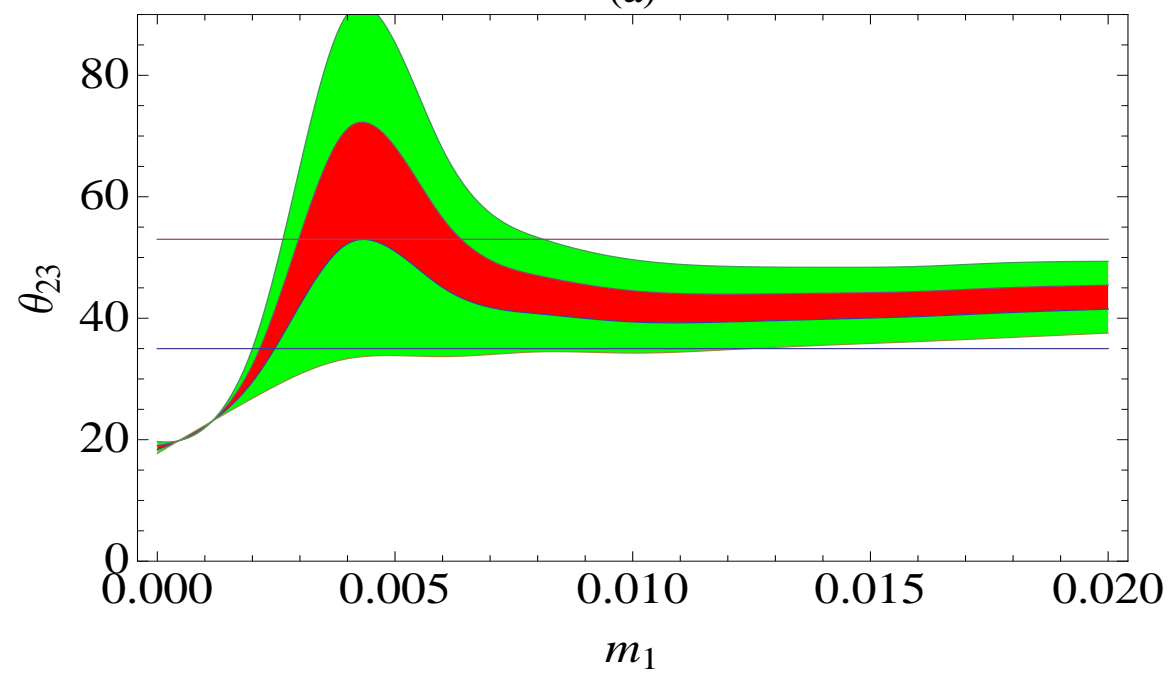

(b)

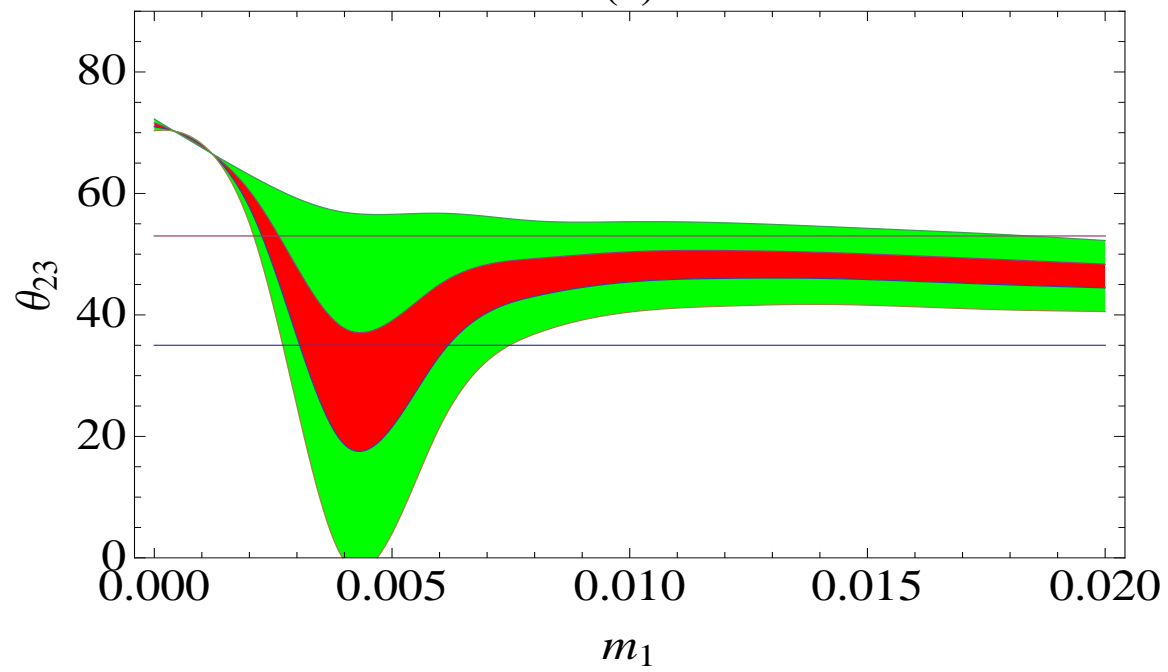

Figure 5: The variation of $\theta_{23}$ with $m_{1}$ for $\alpha=90$ degree for the classes (a) $A_{1}$ and (b) $A_{2}$. The central (red) region is at $1 \sigma$ and the outer (green) region is at $3 \sigma$. 